\title{
Occupational Exposure as it Relate to Pathogenic contamination in some Individuals in some parts of Plateau State, North Central Nigeria
}

\author{
V.A.Pam, D.A.Dakul, S.I.Bata ${ }^{3}$, Obalisa, $^{3}$, Akut $\mathrm{K}^{4}$.Hassan,A.A ${ }^{5}$. K.I. Ogbu ${ }^{3}$ \\ ${ }^{I}$ Department of Parasitology, FCAH AND PT,NVRI,Vom \\ 2Department of Zoology University of Jos \\ ${ }^{3}$ Deparment of Animal Health, FCAH AND PT,NVRI,Vom \\ ${ }^{4}$ Deparment of Extension,FCAH AND PT,NVRI,Vom \\ ${ }^{5}$ College of Act and Science,Plateau State.
}

\begin{abstract}
This study investigated Cryptosporidium species in Apparently Healthy Individuals (AHI) of different occupation the study was carried out in Jos metropolis of Plateau State, Nigeria. Three hundred (300) samples were collected from Apparently Healthy Individuals (AHI). Samples were analyzed for Cryptosporidium infectious. Cryptosporidium species and other intestinal parasites were determined by parasitological techniques (Formol-ether and floatation concentration techniques Staining was done using Zielh Neelsen technique). Cryptosporidiosis in AHI was 37(12.3\%). Younger individuals between the ages of 0-10 and 11-20, had 11(55.0\%), $12(60.0 \%)$ and $15(57.7 \%), 14(58.33 \%)$ in the male and female group. These were statistically $(p<0.05)$ significant. Entamoeba histolytica, Giardia intestinalis, Taenia saginata, Ancyslostoma duodenale, (Hookworm sp) Ascaris lumbricoides, and Hymenolepis nana were also identified. Of these, two major parasites implicated in gastroenteritis and diarrhea (Entamoeba histolytic and Giardia intestinalis) had prevalence of $40(13.33 \%)$ and $11(3.67 \%)$ respectively. The occurrence of this parasite in relation to the occupational group sampled showed that the prevalence was higher with the herdsmen, butchers and the farmers having $17.11 \%, 16.96 \%$ and $15.84 \%$ in that order with the least occurring in the businessmen with $8.33 \%$. These results are discussed in relation to the distribution and significance of Cryptosporidium species and other enteric parasites in the environment.
\end{abstract}

Keywords: - Cryptosporidium species, Individuals, Nigeria, Occupational, Plateau State

\section{INTRODUCTION}

The genus Cryptosporidium is composed of protozoan parasites that infect epithelial cells in the microvillus border of the gastrointestinal tract of all classes of vertebrates. They are found worldwide. Effects of infection vary with the species of Cryptosporidium species. Some species of Cryptosporidium species infect many host species whereas others appear restricted to groups such as rodents or ruminants and still others are known to infect only one host species. Some species primarily infect the stomach whereas others primarily infect the intestine. Some species are pathogenic, whereas the presence of others has not been shown to be related to any disease manifestations. Some infections are acute and self-limiting whereas others are chronic [1],[2]. The severity and duration of infection with pathogenic species are also affected by the immune status of the infected person or animal. Immune competent individuals might suffer mild, moderate, or severe acute illness whereas immunocompromised individuals can suffer severe chronic illness and even death [3].The ability of Cryptosporidium species to infect humans and a wide variety of animals and because of the ubiquitous presence of Cryptosporidium oocysts in the environment, humans can acquire Cryptosporidium species infections through several transmission routes[4]. These include direct person-to-person or animal-to-person transmission and indirect waterborne and food borne transmission, and the parasites can be of anthroponotic or zoonotic origin. The role of each transmission route in endemic areas, however, is frequently unclear because of the expensive nature of epidemiologic investigations and the inability to differentiate Cryptosporidium species by conventional microscopy.

There are now sixteen recognized species and nearly triple this number of unnamed cryptosporidians that have been identified only as genotypes. However, most studies on the biology, morphology, biochemistry, host preferences, immunology, pathogenicity, physiology, and prevalence have been conducted on one species, Cryptosporidium parvum. The reason: this species is of medical and veterinary importance, it is geographically widespread, and it infects many host species producing prodigious numbers of oocysts making it more easily obtainable for study than other species of Cryptosporidium species and it can be grown and tested in vitro and in animal models. Consequently data derived from Cryptosporidium parvum, in some cases, have become generalized 
and extended to other members the genus. Obviously each species and genotype has individual characteristics that make it different from the others.

Cryptosporidiosis in humans is predominantly a diarrheal disease with the organisms growing in an intracellular but extra-cytoplasmic location in the enterocytes of the gastrointestinal tract. Infection affects all age groups and can occasionally affect other organs. The disease is more protracted, severe and affects extraintestinal sites in people with innate or acquired deficiencies in immunity[5], [6].

Cryptosporidium species causing symptomatic disease was first noted in turkeys in 1955. During the 1970s Cryptosporidium species infections were reported to cause neonatal diarrhea in calves [7], [8]. The first human cases of cryptosporidiosis in humans were recorded in the 1970s: one in a young girl with enterocolitis [8]. With the developing AIDS pandemic in the 1980s more cases of cryptosporidiosis in AIDS were identified [9] and Cryptosporidium was found to be a cause of diarrhea in immunocompetent people [6], [5]. Cryptosporidiosis occurs worldwide, Sero-epidemiological studies of particular areas have indicated that the percentage of the population affected at some time in life can vary from under $20 \%$ to over $90 \%$ [10].

\subsection{Study Area}

\section{MATERIALS AND METHODS}

The study was carried out in one Local Government Area of Plateau State, namely the Jos North Local Government Areas (JNLGA). Ethical clearance was process from the management of Faith Alive Foundation before the commencement of this research. Three hundred (300) stool samples were collected from the apparently healthy. Samples were collected based on the different age categories of the subjects sampled. Questionnaires, verbal communication were used to get other information from the subject.

\subsection{Collection of Samples}

Human stool samples were collected in properly labeled sterile wide-mouthed plastic bottles, most of which were collected the next day but few were collected on the same day. Demographic information, (occupation) history and observable clinical conditions of each individual were recorded along with description of stool (watery, loose, or well formed, mucoid, bloody and coloured). The samples were then transported in Coleman icebox to the Parasitology and Diagnostic Laboratory of the National Veterinary Research Institute, Vom, Nigeria for further analysis. Processing of samples commenced immediately but all unprocessed samples were preserved in $10 \%$ formalin solution and refrigerated all analysis was done within a week.

\subsection{Processing of Faecal Samples}

Feacal samples were screened using two methods. The formal ether concentration and the saturated sodium chloride floatation techniques. The McMaster counting technique was used for counting the frequency of occurrence of the oocysts following the manufacturer's instructions. Oocysts of Cryptosporidium species can be detected in wet preparations but they are more easily identified in smear, stained by the modified ZiehlNeelsen (Zn) method following concentration by the formol ether oocysts concentration technique as described by the manufacturer.

\section{RESULTS AND DISCUSSION}

The study population was 300 individuals, who were apparently healthy (AH) 37 (12.33\%). Other enteric parasites encountered in the healthy group were Entamoeba histolytica 40(13.33\%), Ancyslostoma duodenale 23(7.67\%), Giardia intestinalis and Hymenolepis nana each with 11(3.67\%). the subjects had low prevalence of the infection $12.33 \%$ of Cryptosporidium sp. and 3.67\% of Giardia intestinalis (Table 1).

Table 1: Prevalence of Cryptosporidium species and other Parasites in Human

\begin{tabular}{lll}
\hline Parasites & No. $+\mathrm{ev}$ & Percentage \\
\hline Cryptosporidium sp. & 37 & 12.33 \\
Giardia intestinalis & 11 & 3.67 \\
Entamoeba histolytica & 40 & 13.33 \\
Hymenolepis nana & 11 & 3.67 \\
Taenia saginata & 16 & 5.33 \\
Ascaris lumbricoides & 16 & 5.33 \\
Ancylostoma duodenale & 23 & 7.67 \\
TOTAL & $\mathbf{1 5 4 . 0 0}$ & $\mathbf{5 1 . 3 3}$ \\
MEAN & $\mathbf{2 2 . 0 0}$ & \\
SD & $\mathbf{1 2 . 0 0}$ & \\
\hline
\end{tabular}




\subsection{Distribution of Cryptosporidium species according to Occupational groups.}

From Figure 1, there exist differences in the distribution of this parasite in the different occupational groups sampled. High prevalences were observed with the classes of occupation that have direct contact with the animals and their faecal matter: Butchers 19(16.96\%), Famers 16 (15.84\%), Herdsmen 13(17.11\%) when compared to the groups of occupation that just occasionally visit the animal market or the abattoir: civil servants $6(7.06 \%)$, businessmen $8(8.33 \%)$ and veterinary workers, $4(8.70 \%)$.

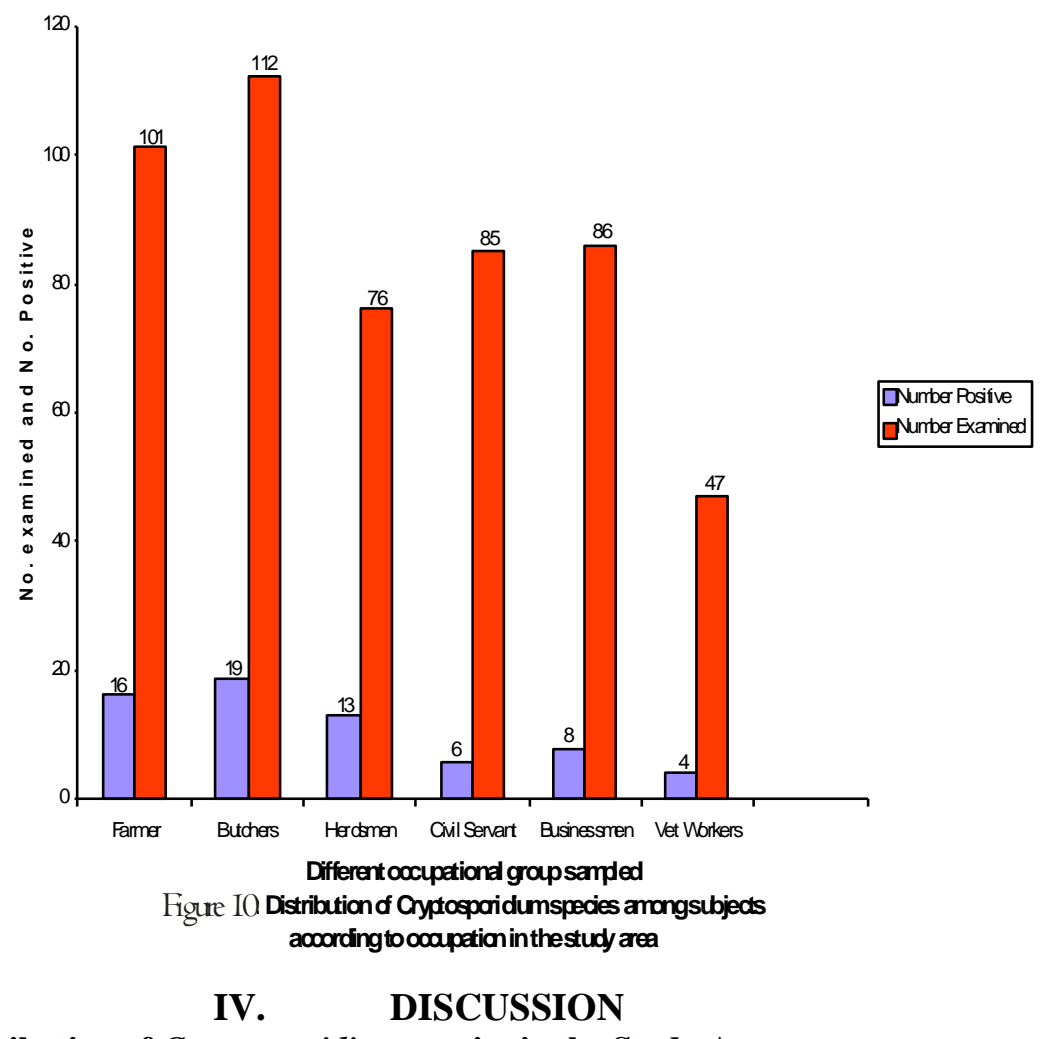

\subsection{Occupational Distribution of Cryptosporidium species in the Study Area.}

Our finding shows a positive correlation $(\mathrm{p}<0.05)$ between Cryptosporidium species prevalence and peoples occupations. Prevalence of Cryptosporidium sp was high in butchers (30.64\%), followed by the farmers (25.80\%) and herdsman (20.90\%) the least prevalence was with the veterinary workers $(4.17 \%)$. (3) in a study to assess the occupational risk of Cryptosporidium species infection in Tiledo reported fourteen out of the 16 sites positive for Cryptosporidium species (77.8\%),occupational exposure to Cryptosporidium species is very plausible. On a similar note, [11] and [3] also reported prevalence of $18.2-25.6 \%$ with the farmers and the herdsmen. The relatively high prevalence among farmers and the herdsmen could be attributed to frequency of exposure to cryptosporidiosis, faecal contamination through water, manure. Our results in this study support the fact that economic condition of individuals is a risk factor in Cryptosporidium infection [12].

On the contrary [1] reported low prevalence (1.1\%) of Cryptosporidium in butchers. However, [12] and [9],[1] and [11] reported lower prevalence of (1.1to $2.0 \%$ ) in the butchers' and farmers from developed countries(USA, and the UK). Water treatment does not prevent faecal oral contact, an event that is likely responsible for the majority of the transmission of these parasites among herd members .Consumption of water from field ponds during spring runoff could also negate any benefits that water treatment has on reducing water-borne transmission of these parasites other sources of transmission can be exposure to infected animals and humans [12], [5].

The lowest prevalence was with the Vet workers $(4.17 \%)$,this can be as a result of their level of enlightenment which makes them cautious [10], [12]. Educational visit to working farms during the lambing season can also be a source of contamination [13]. 


\section{REFERENCES}

[1] Ciçek M, Korkoca H, Gül A. Investigation of Cryptosporidium sp. in workers of the Van municipality slaughterhouse and in slaughtered animals. Livestock2006; 102( 3): 204-210.

[2] Graczyk, TK..andCranfied, MR. (1998). Zoonotic transmission of Cryptosporidium: Implication for water-borne Cryptosporidiosis. Parasitology Today 13(9): 348 - 350.

[3] Hailu, K, Brian,H,Michael, S.Risk of Occupational Exposure to Cryptosporidium, Giardia, and Campylobacter Associated with the Feces of Giant Canada Geese .Applied Occupational and Environmental Hygiene, 2001;16: (9) 905 - 909.

[4] Mansfield, LS and Gajadhar, AA. Cyclosporacayetanensis, a food and water borne coccidian parasite. Veerinary Parasitology 2004; 126, 73-90.

[5] Moon, HW. andWoodmansee, DB. Cryptosporidiosis Zoonosis updates. Journal of American Veterinary Medical Association. 1986;189(6): 643-646.

[6] O’Donoghue, PJ. Cryptosporidium and cryptosporidiosis in man and animal. International Journal of Parasitology 1995;25: 139- 195.

[7] Pohjola, S. Jokipii, AMM and Jokipii, L. Sporadic Cryptosporidiosis in a rural population is asymptomatic and associated with contact to cattle. Acta Veterinary Scandinavica1986a; 27: 91 -102

[8] 13. Poonacha, KB. and Pippin, C. Intestinal cryptosporidiosis in a cat. Veterinary Pathology 1982; 19: $708-710 \mathrm{C}$

[9] Reese, NC., Current, WL., Ernsts, JV., and Bailey, WS. Cryptosporidiosis of man and calf: A case report and results of experimental infections in mice and rats. American Journal of Tropical Medicine and Hygiene 1982; 31: 2226 - 229.

[10] Suleiman, MM. Cryptosporidiosis in humans: A review. Nigerian Journal of Parasitology 2002; 23(1): 83-93.

[11] Smith, HV., Girdwood, RWA., Peterson, WJ., Hardle, R., Green, LA., Benton, C., Tulloch, W., Sharp, JCM. and Forbes, GI. Water-borne outbreak of Cryptosporidiosis (Letter) Lancetii: 1988; 1484.

[12] Tzipori, S., Angus, KW., Gray, EW. and Campbell, I.. vomiting and diarrhea associated with Cryptosporidial infections (Letter). The New England Journal of Medicine 1980b; 3: 8.

[13] Xiao ,LH, Herd, RP, Rings, DM. Concurrent infections of Giardia and Cryptosporidium on two Ohio farms with calf diarrhea. Veterinary Parasitology. (1993). 51:41-48. 